\title{
An On-Axis Tomography Holder for Correlative Electron and Atom Probe Microscopy
}

Approved for public release. Distribution is unlimited.

Philip D. Edmondson

Date: August 30, 2017 


\section{DOCUMENT AVAILABILITY}

Reports produced after January 1, 1996, are generally available free via US Department of Energy (DOE) SciTech Connect.

Website: http://www.osti.gov/scitech/

Reports produced before January 1, 1996, may be purchased by members of the public from the following source:

National Technical Information Service

5285 Port Royal Road

Springfield, VA 22161

Telephone: 703-605-6000 (1-800-553-6847)

TDD: $703-487-4639$

Fax: 703-605-6900

E-mail: info@ntis.fedworld.gov

Website: http://www.ntis.gov/help/ordermethods.aspx

Reports are available to DOE employees, DOE contractors, Energy Technology Data Exchange representatives, and International Nuclear Information System representatives from the following source:

Office of Scientific and Technical Information

PO Box 62

Oak Ridge, TN 37831

Telephone: 865-576-8401

Fax: 865-576-5728

E-mail: report@osti.gov

Website: http://www.osti.gov/contact.html

This report was prepared as an account of work sponsored by an agency of the United States Government. Neither the United States Government nor any agency thereof, nor any of their employees, makes any warranty, express or implied, or assumes any legal liability or responsibility for the accuracy, completeness, or usefulness of any information, apparatus, product, or process disclosed, or represents that its use would not infringe privately owned rights. Reference herein to any specific commercial product, process, or service by trade name, trademark, manufacturer, or otherwise, does not necessarily constitute or imply its endorsement, recommendation, or favoring by the United States Government or any agency thereof. The views and opinions of authors expressed herein do not necessarily state or reflect those of the United States Government or any agency thereof. 
Materials Science and Technology Division

\title{
AN ON-AXIS TOMOGRAPHY HOLDER FOR CORRELATIVE ELECTRON AND ATOM PROBE MICROSCOPY
}

\author{
Philip D. Edmondson
}

Date Published: August 30, 2017

Work Package Title: FY17 ORNL Infrastructure Award

Work Package \#: UF-17OR020505

Work Package Manager: Philip D Edmondson

Milestone \#: M3UF-17OR0205054

Prepared by

OAK RIDGE NATIONAL LABORATORY

Oak Ridge, TN 37831-6283

managed by

UT-Battelle, LLC

for the

US DEPARTMENT OF ENERGY

under contract DE-AC05-00OR22725 



\section{CONTENTS}

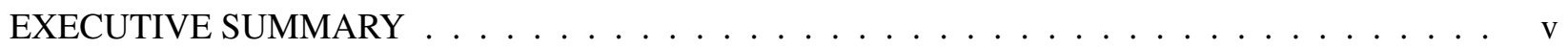

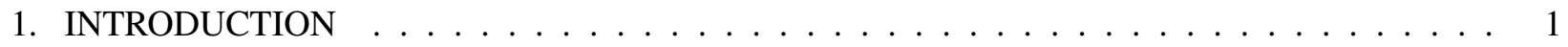

1.1 Facilities and Resources . . . . . . . . . . . . . . . . . . . . 1

1.1.1 Low Activation Materials Development and Analysis (LAMDA) Laboratory . . . . . 1

1.1 .2 FEI Talos F200X STEM . . . . . . . . . . . . . . . . . . . . 1

1.1.3 Atom Probe Tomography . . . . . . . . . . . . . . . . . . . . . 2

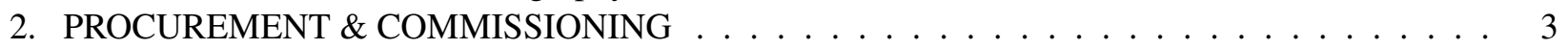

2.1 Notes on Sample Preparation . . . . . . . . . . . . . . . . . . . . . 5

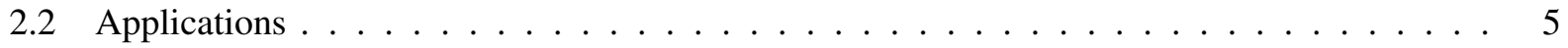

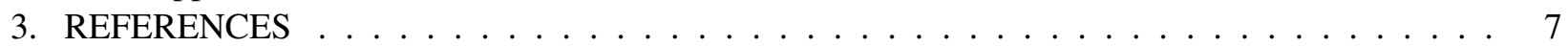





\section{EXECUTIVE SUMMARY}

This document briefly outlines the procurement and commissioning of a Fishione Model 2050 on-axis rotational tomography specimen holder for the FEI F200X Talos Scanning Transmission Electron Microscope in Oak Ridge National Laboratory's Low Activation Materials Development and Analysis

(LAMDA) Laboratory. The Model 2050 specimen rod was successfully procured and commissioned and is now available to users. 



\section{INTRODUCTION}

This brief report documents the procurement, receipt, and potential applications of a Fischione Model 2050 on-axis rotation tomography holder that allows for specimens to the examined through $360^{\circ}$ in a transmission electron microscope (TEM). This specimen holder was specifically procured for use with the FEI F200X Talos Scanning/Transmission Electron Microscope (STEM) in Oak Ridge National Laboratory's (ORNL's) Low Activation Materials Development and Analysis (LAMDA) Laboratory.

Not only with the Fischione on-axis tomography holder allow for tomographic data to be obtained from a materials specimen, but it will also facilitate correlative microscopy studies combining STEM imaging, with chemical analysis via energy dispersive X-ray spectroscopy (EDS), and post STEM-EDS analysis atom probe tomogrpahy data collection and analysis. Such a holistic materials characterization on the same specimen volume will further irradiated materials science knowledge.

\subsection{Facilities and Resources}

\subsubsection{Low Activation Materials Development and Analysis (LAMDA) Laboratory}

The LAMDA Laboratory is a world-class, multipurpose irradiated materials science facility for evaluation of materials with low radiological threat. It consists of four laboratory suites containing specialized instruments for materials testing and characterization. The LAMDA facility typically allows for the examination of low or no radioactivity samples $(<100 \mathrm{mR} / \mathrm{hr}$ at $30 \mathrm{~cm})$ without the need for remote manipulation. The preparation facilities for small and compact samples allow researchers to leverage cutting-edge characterization and testing equipment for studying radiation effects in materials. The most commonly conducted work includes mechanical testing, optical and electron microscopy, atom probe tomography, densitometry, metallography, thermal and electrical conductivity. New or infrequently performed activities are also possible with appropriate planning.

The LAMDA facility is utilized by several programs within ORNL's Materials Science and Technology Division, with primary emphasis on the evaluation of irradiated materials. Current programs include the US Department of Energy, the Naval Reactor Advanced Structural Materials Program, the NE-Generation IV program, as well as international nuclear companies and research laboratories. The LAMDA facility is also a part of the ATR NSUF and hosts PhD and post-doctoral researchers on a routine basis.

\subsubsection{FEI Talos F200X STEM}

An FEI Talos scanning/transmission electron microscope (STEM) is located in the LAMDA Laboratory suite of electron optical instruments. This microscope is specifically geared towards the structural and chemical characterization of materials due to the integration of the FEI "SuperX" four- sensor, large solid-angle X-ray detector combined with its high-resolution probe size down to $1.6 \AA$. Furthermore, up to four different imaging signals can be simultaneously obtained using the high angle annular dark field, two annular dark field, and one bright field detector (in STEM mode). Additionally, the Ceta ultra-fast TEM camera ( 25 frames per second) is uniquely suited to capturing in situ experiments such as those that may be conducted with the Protochips Fusion system. 
This instrument is a world-class, atomic-scale structural and chemical characterization with unique capabilities in order to conduct leading experiments on nuclear materials, e.g. [1]

\subsubsection{Atom Probe Tomography}

Atom Probe Tomography (APT) is a technique capable of providing highly spatially and chemically resolved three-dimensional atom-by-atom rendering of a material specimen. [2] The development of laser pulsing enables the analysis of significantly less conductive specimens enabling a wider range of materials to be analyzed. The combination of laser assisted APT and the development of robust specimen preparation techniques based on focused ion beam (FIB) $[3,4]$ have revolutionized the technique, not only enabling the analysis of poorly conducting specimens, but also site-specific sample fabrication, particularly from ion-irradiated samples. [4] The unique traits of APT are the three-dimensionality of the analysis and the capacity to resolve the chemical identities of atoms, even at extremely dilute concentrations or of very light species that are not easily distinguishable via electron microscopy based techniques, making correlative microscopy using STEM-EDS and APT techniques highly desirable.

No atom probe is available within the LAMDA laboratory, but access can be obtained for the NSUF laser assisted atom probe at the CAES facility. 


\section{PROCUREMENT \& COMMISSIONING}

A Fischione Model 2050 on-axis rotation tomography holder was procured and delivered in Fiscal Year 17 (FY17). A photograph of the holder within its storage case is shown in Figure 1. Another photograph showing the specimen rod on the sample loading mount is shown in Figure 2.

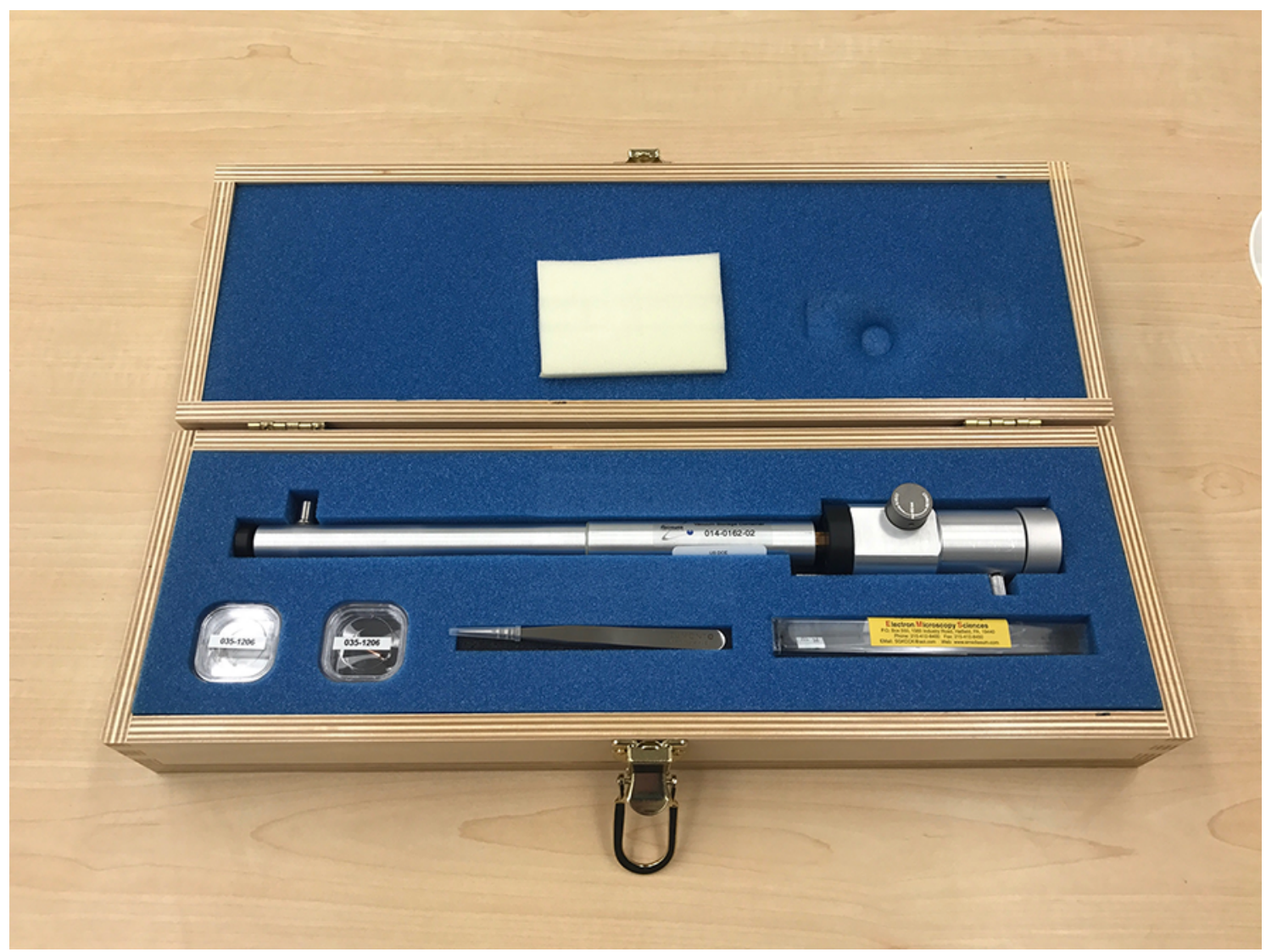

Figure 1. Photograph showing the Fischione Model 2050 on-axis rotation tomography holder in its storage case. Also visible are the specimen mounts and tweezers required for specimen exchanges.

To mount and load specimens, a sample is prepared via FIB methods following the protocol for APT specimens. [3, 4] This is done as the APT specimen geometry provides the idea specimen for on-axis rotational tomography, and allows for APT to be conducted on the specimen following STEM-EDS rotational tomography data collection. The samples prepared by FIB are then landed on a specimen mount. In the Model 2050 kit, small copper tips are provided for this purpose. First experiments conducted using this holder utilized these tips. Following removal of the specimen and tip from the FIB, the sample-tip combination is loaded into the specimen rod using the mount shown in Figure 2. To protect the specimen during loading into the microscope, the specimen rod has the capability to retract the sample-tip into the holder itself as shown in Figure 3

A sample was mounted onto the $\mathrm{Cu}$ tip and the specimen rod and loaded into the FEI F200X Talos instrument to ensure correct operation of the rod and that no vacuum leaks were present. This was done successfully and the specimen rod was determined to be available for use. It should also be noted that this 


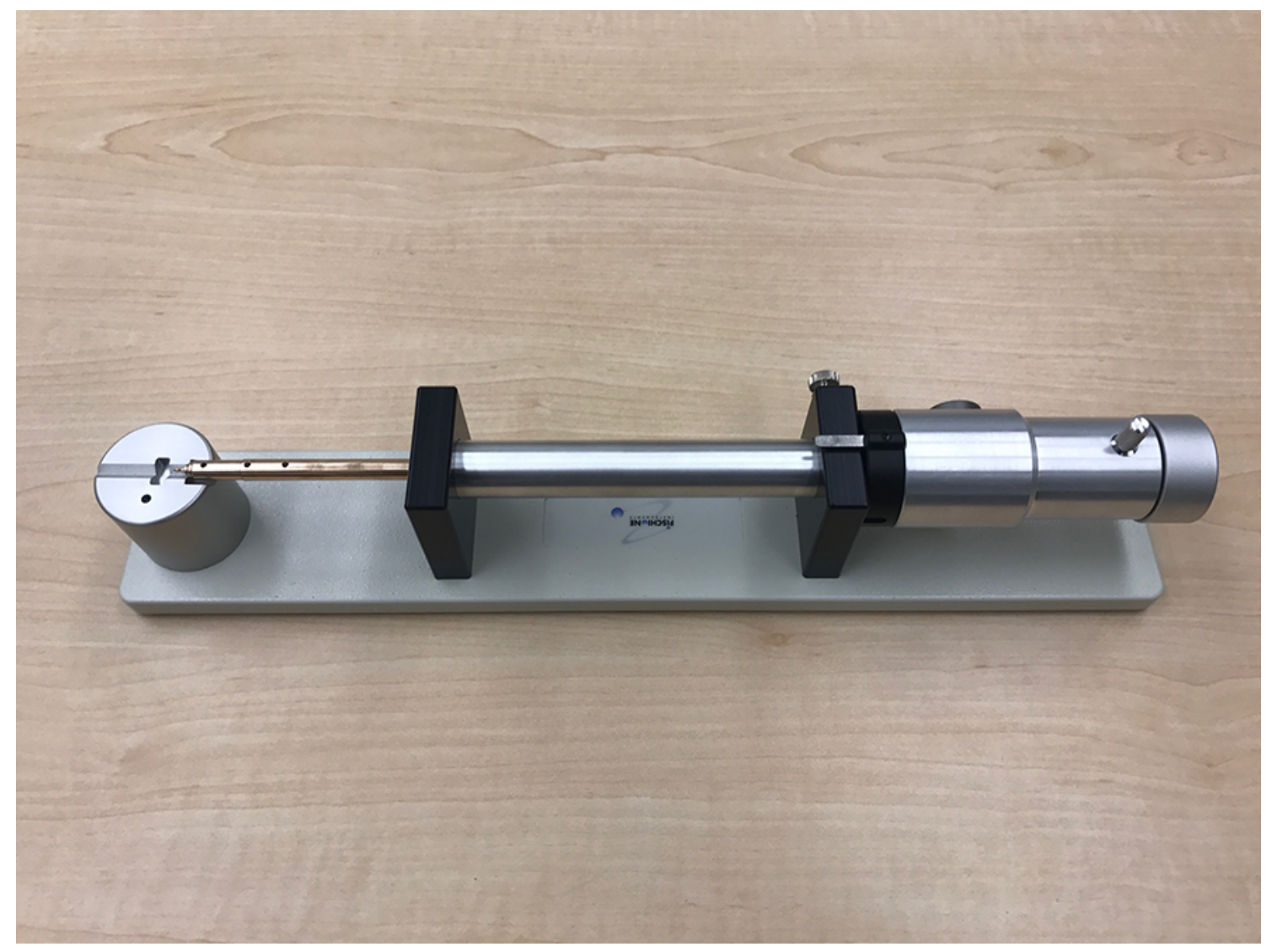

Figure 2. Photograph showing the Fischione Model 2050 on-axis rotation tomography holder on the mounting unit that allows for specimen exchanges to be performed. 


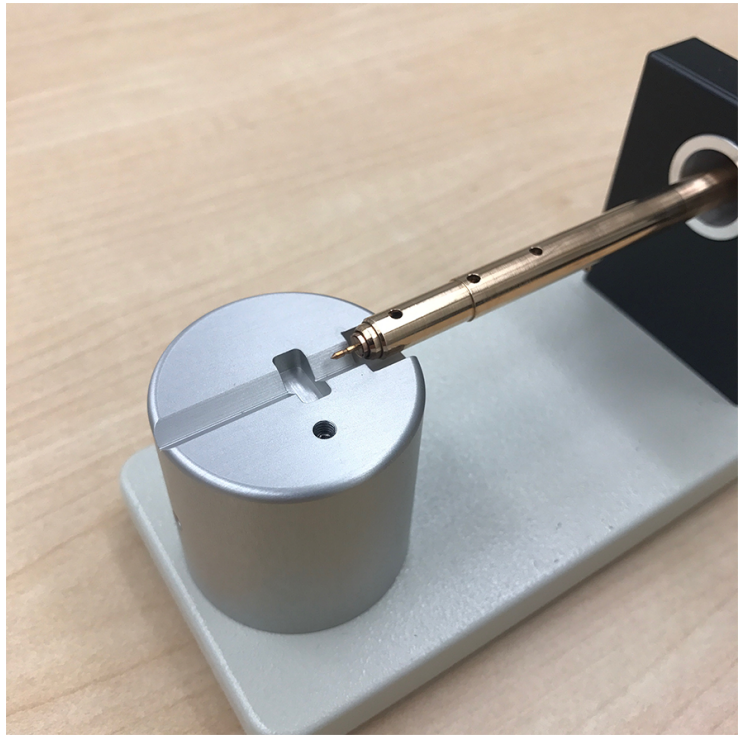

(a)

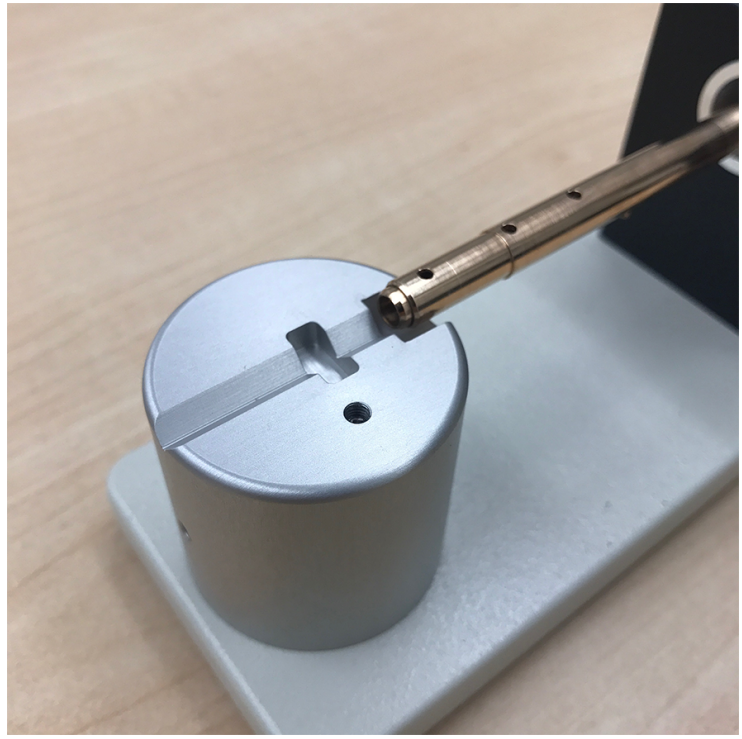

(b)

Figure 3. Photographs showing a) the sample-tip in the extended position ready for mounting of the specimen; b) the sample-tip int he retracted position ready for insertion into the TEM.

is a purely mechanically driven system with no electronics required.

\subsection{Notes on Sample Preparation}

During the initial specimen preparation and subsequent imaging, it was noted that the $\mathrm{Cu}$ tip had a very large landing base area (approximately $250 \mu \mathrm{m}$ in diameter). It is anticipated that this could cause some issues when performing correlative STEM-EDS/APT analysis due to reduced field enhancement at the tip during APT data acquisition. As such, a solution whereby tungsten wires were electropolished to a fine point then mounted in $\mathrm{Cu}$ tube as per standard APT electropolishing protocols. Following this initial preparation, the specimen of interest could be mounted on this small radius of curvature tungsten wire thereby maintaining high fields in the atom probe.

\subsection{Applications}

The applications for this holder are numerous: it will allow for three-dimensional analysis to be conducted on two different analytical instruments offering the advantages and strengths of both techniques to be summed in order to obtain highly valuable data. This could include the examination of grain boundaries in more detail, with a particular focus on depleted regions about the boundaries. More examples and practical considerations can be found in [5]. 


\section{Acknowledgments}

The purchase of this specimen holder was made possible by funding from the Department of Energy's Nuclear Science User Facilities (NSUF) program, under Infrastructure award UF-17OR020505. FEI Talos F200X STEM provided by the Department of Energy, Office of Nuclear Energy, Fuel Cycle R\&D Program and the Nuclear Science User Facilities.

\section{REFERENCES}

\section{References}

[1] P.D. Edmondson, C.M. Parish, and R.K. Nanstad. Using complimentary microscopy methods to examine Ni-Mn-Si-precipitates in highly-irradiated reactor pressure vessel steels. Acta Materialia, 134:31-39, 2017.

[2] T. F. Kelly and M. K. Miller. Invited review article: Atom probe tomography. Review of Scientific Instruments, 78(3):1-20, 2007.

[3] M. K. Miller and K. F. Russell. Atom probe specimen preparation with a dual beam SEM/FIB miller. Ultramicroscopy, 107(9):761-766, 2007.

[4] M. K. Miller and Yanwen Zhang. Fabrication and characterization of APT specimens from high dose heavy ion irradiated materials. Ultramicroscopy, 111(6):672-675, 2011.

[5] Wei Guo, Brian T. Sneed, Lin Zhou, Wei Tang, Matthew J. Kramer, David A. Cullen, and Jonathan D. Poplawsky. Correlative Energy-Dispersive X-Ray Spectroscopic Tomography and Atom Probe Tomography of the Phase Separation in an Alnico 8 Alloy. Microscopy and Microanalysis, 22(06):1251-1260, 2016. 